\title{
Application of the Fuzzy Computational Intelligence in Power Quality Data Management
}

\author{
Dr. Hoda Farag \\ Alexandria Electricity Distribution Company, Alexandria, Egypt \\ Hoda_farag77@yahoo.com
}

\begin{abstract}
In Electrical Power Distribution System the sustained availability and quality of electric power are the main challenge they need to satisfy so overcoming the power quality $(\mathrm{PQ})$ degradation became an asset. This Paper addresses the perfect load management using the computational techniques by analyzing the data of the system taking into account the density of the feeding nodes and its distribution also the classification of major Power quality degradations such as power factor and harmonics in the System and The methodology will be illustrated, simulated and evaluated using the fuzzy technique clustering the data and on an Artificial Neural Network (ANN) to achieve the optimum utilization of the energy loads and perfect load management and optimization. Simulation results demonstrate the effectiveness of the proposed algorithm in reducing the power and energy losses, improving the quality of the electric power system.
\end{abstract}

Keywords - Power Quality Degradaion; fuzzy technique; Artificial Neural Network.

\section{INTRODUCTION}

The power quality is an important issue to maintain the stability of the Electric Power Distribution System (EPDS) and its instability due to any kind of disturbance leads to malfunction of the end user devices or the complete outage in the Energy distribution system.

The degradation in quality of power is mainly caused by disturbances such as voltage swell, voltage sag, notch, transients, and harmonic distortions and mainly the non-linear loads that draw harmonic currents, and as a consequence, harmonic voltages are generated whenever the harmonic current flows through the impedance of the system which are the main cause of power quality (PQ) degradation leading not only to the (PQ) degradations or temporary interruption but also to ultimately complete outage of EPDS[1].

The power quality became more important to maintain the power system and so the exact and fast detection of the disturbances created the need to have a precise system that detect the time and duration of the occurrence of the disturbance analyzing the data of the $P Q$ field and classifying them according to the rate of occurrence and their effect on the Quality of the network.

Soft computing techniques like the fuzzy logic, neural networks or combination of them are used to design an intelligence system. The fuzzy logic used for reasoning while neural networks provide algorithms for learning, classification, and optimization [2], although there are substantial areas of overlap between neural networks, FL, and probabilistic reasoning.

Modern spectral and harmonic analysis is based on Fourier based transforms. However, these techniques are less efficient in tracking the signal dynamics for transient disturbances. Consequently, The wavelet transform has been introduced as an adaptable technique for non-stationary signal analysis. Different power quality disturbances are classified by a unique energy distribution pattern based on the difference of the discrete wavelet coefficients of the analyzed signal and a pure sine wave. [4]. Also the S-transform algorithm and continuous wavelet transform (CWT) are time frequency algorithms powerful in detection and classification of $\mathrm{PQ}$ disturbances as in [5] comparing the effect of each on different categories of disturbances. As proposed in [6] the power quality disturbance recognition system contain 
three main components which are a simulator to generate the power quality disturbances and the detector which used the DWT to detect the power signal disturbances and the neural network architecture to classify the disturbances with increases the accuracy of detection.

In this paper we propose a technique that analyzes the $P Q$ data of the system under consideration Taking into account the Power factor and peak hour table in EPDS to predict the undesired PQ disturbance in the system. This will provides power system engineers to formulate intelligent strategy for efficient power system operations. The proposed algorithm incorporates multi-band wavelet transformation and the modern computation intelligent approaches like fuzzy technique and the artificial neural network.

This paper is organized as follows In the first section we present a review of related work, In Section 2 we clarify different sources of major power degradations like the power factor and the harmonics and their classification, In Section 3 presents some fundamental Techniques used in our algorithm like the fuzzy technique. Section 4,we describe the proposed algorithms for the power quality disturbance detection. Section 5 , we report the effectiveness of our method and compare results of each algorithm used; At last conclusion of this paper will be drawn in Section 6 .

\section{CLASSIFICATION OF MAJOR POWER QUALITY DEGRADATION}

The power quality term $P Q$ can be defined as voltage quality with reference to slow variation in voltage magnitude and to indicate the existence of an adequate and secure power supply.

The patterns of voltage or $P Q$ disturbances need to be identified for preventive action in order to avoid the problem in the electrical system and the cause of any deviation from the ideal waveform. $P Q$ disturbances/events cover a broad frequency range with significantly different magnitude variations and can be non-stationary, thus, accurate techniques are required to identify and classify these disturbances [3].

- Most of the PQ problems fall into those two basic categories:-

\section{A. Non steady state variation}

which is the abnormality in the voltage or the current Transient voltages may be detected when the peak magnitude exceeds a specified threshold. RMS voltage variations (e.g., sags or interruptions) may be detected when it exceeds a specified level. This is classified according to the nature of the waveform distortion. For non steady state disturbances, other attributes such as rate of rise, rate of occurrence and energy potential are useful.

\section{B. Steady-State Variations}

Steady state variation is basically a measure of the magnitude by which the voltage or current may vary from the nominal value, plus distortion and the degree of unbalance between the three phases. These include normal rms voltage variations, and harmonic and distortion. For steady-state disturbances, the amplitude, frequency, spectrum, modulation, source impedance, notch depth and notch area attributes can be utilized.

\section{- Power factor}

The power factor is (P.F) is a relation between the actual power and the apparent power as given by equation (1)

$$
P . F=\cos \Phi
$$

The voltage unbalance contributes to poor power factor in EPDS with the increase of non-linear loads also as a result of the voltage unbalance between the three phases.

As most of the distribution companies impose a penalty to its customers if THEIR loads contributes towards poor power factor so we need to monitor the power factor at the industrial customers to force them to take all adequate steps to maintain their power factor close to unity.

\section{- Harmonics}

Harmonic distortion is in the voltage and the current waveforms in power distribution networks as in equations 2 and 3 where $\mathrm{Vh}$ and $\mathrm{lh}$ are the amplitude of the voltage and the current at the harmonic component and Vrms and Irms are the root mean square values of voltages and currents for the non-sinusoidal waveforms [9] 


$$
\begin{aligned}
& V_{m s}=\sqrt{\sum_{t=1}^{m_{m a x}}\left(\frac{1}{\sqrt{2}} V_{k}\right)^{2}}=\frac{1}{\sqrt{2}} \sqrt{\left(V_{1}^{2}+V_{2}^{2}+V_{3}^{2}+. .+V_{t_{\max }}^{2}\right.} \\
& I_{m s}=\sqrt{\sum_{h=1}^{h_{\max }}\left(\frac{1}{\sqrt{2}} I_{h}\right)^{2}}=\frac{1}{\sqrt{2}} \sqrt{\left(I_{1}^{2}+I_{2}^{2}+I_{3}^{2}+. .+I_{h_{m}}^{2}\right.}
\end{aligned}
$$

The total harmonic distortion (THD) is a measure of the harmonic equipment in a distorted wave as in equation 4.

$T H D=\frac{\sqrt{\sum_{h>1}^{h_{\max }}\left(M_{h}\right)^{2}}}{M_{1}}$

Where $M_{h}$ is the root mean square value of the harmonic component.

The power Distribution network is ideally designed to tackle sinusoidal voltage and current waveforms and the existing power distribution network design is only capable of absorbing harmonic distortion to a certain limit. [10] And the extensive use of the nonlinear loads that the conventional power system not designed to accept it that increases the power quality disturbances so we need fast and intelligent methods to detect theses disturbances like the methods will be mentioned later.

\section{FUZZY LOGIC SYSTEM}

The Fuzzy logic poses the ability to mimic the human mind to effectively employ modes of reasoning that are approximately rather than exact especially in decisions or action that are based on precision and certainty [7] .

Zadeh [11] introduced the term fuzzy logic which described the mathematics of fuzzy set theory (1965) indicating that there was a third region beyond True and False by declaring the fuzzy set that allows partial membership which is an extension of a crisp set that allows only full membership or no membership at all.

In designing the fuzzy inference system, membership function is essentially the curve that defines how each point in the input space is mapped to a membership value (or degree of membership) between 0 and 1 . Various types of membership functions are used, including triangular, trapezoidal, generalized bell shaped, Gaussian curves, polynomial curves, and sigmoid functions and the fuzzy inference systems consist of if-then rules that specify a relationship between the input and the output fuzzy sets .

As shown in Figure 1 the fuzzy inference system (FIS) essentially defines a nonlinear mapping of the input data vector into a scalar output, using fuzzy rules. The mapping process involves input/output membership functions, FL operators, fuzzy if-then rules, aggregation of output sets and defuzzification.

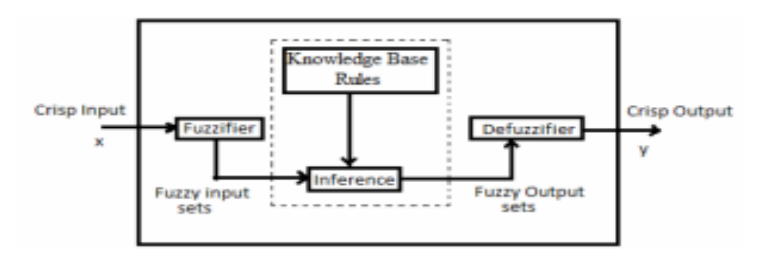

Fig.1. Block Diagram of the Fuzzy Inference system

\section{THE PROPOSED ALGORITHM}

To minimize power quality disturbances and to devise suitable corrective and preventive measures, efficient detection and classification techniques are required in the emerging power systems. As the recent development in the technologies of the signal processing and the artificial intelligence we were able to develop more accurate system based on these new techniques.

The First step is to develop or pick a suitable method for the detection of the $P Q$ Degradations which is the wavelet transforms based on the normentropy based feature extraction which is an ideal tool for non-stationary signals and the classification of theses degradations such as the interruption, the waveform distortions, harmonics, sags, swells, then clustering these data into our system with the power factor measurements and the peak hour table and major power quality degradation like the harmonics using the fuzzy technique and the artificial neural network specifying the rules that enable us to detect the disturbances and hence the perfect optimization of the energy loads.

\section{A. Feature Extraction and Detection of (PQ) Disturbances using DWT}

The Wavelet domain is a promising domain as it is a frequency domain technique. DWT is more preferable for detection of time-frequency variations as it employs a short window for high frequency components and long window for low frequency components which provides the best time frequency resolution to detect the all Power quality disturbances.

The Wavelet transform uses wavelet function as the 
basis function which scales itself according to the frequency under analysis. As it uses the mother wavelet function $\psi$ which will generate the high frequency components (detail) and scaling function $\phi$ which will generate the low frequency components (approximation) to perform simultaneously the multi resolution analysis decomposition and later the reconstruction of the signal.

The wavelet function is defined as follows:

$f(x)=\sum_{i_{i} j} c_{i, j} \Psi_{i, j}(x)$

Where $\psi \mathrm{i}, \mathrm{j}(\mathrm{x})$ are the wavelet expansion function and the two parameter expansion coefficients ci,j are the discrete wavelet transform coefficients of $f(x)$ where $j$ is the translation and $i$ is the dilation parameter

$C_{i j j}=\int_{-\infty}^{5 \infty} f(x) \psi_{i j, j}(x)$

(6)

The mother wavelet is related to the scaling function as follows:
$\Psi(\mathrm{x})$
$=$
$\sqrt{ } 2 \sum g(k)$
$\phi(2 x-k)$

(7)

And

$g(k)=$

$(-1) \mathrm{k}$

$h(1-k)$

(8)

where $g$ and $h$ are the filter coefficients of half- band low-pass and high-pass filters.

As the wavelet transform uses a time- window function that changes in frequency as the mother wavelet is scaled and translated to provide information in the frequency and the time domain not like the STFT where the window function is fixed.

Using the sub band coding in the WT the signal is decomposed into different frequency levels and presented as wavelet coefficients. Depending on the types of signal, continuous wavelet transforms (CWT) or discrete wavelet transform (DWT) are employed.

The waveforms in fig 2 generated using the
MATLAB indicating 6 types of disturbances which are sag, swell, interruption, harmonics, sagharmonics, swell -harmonics.

The statistical parameters used for Power quality disturbance detection are the sample mean and variance and wavelet entropy so comparing the original power signal without disturbance and signal with disturbance the disturbance type can be detected.
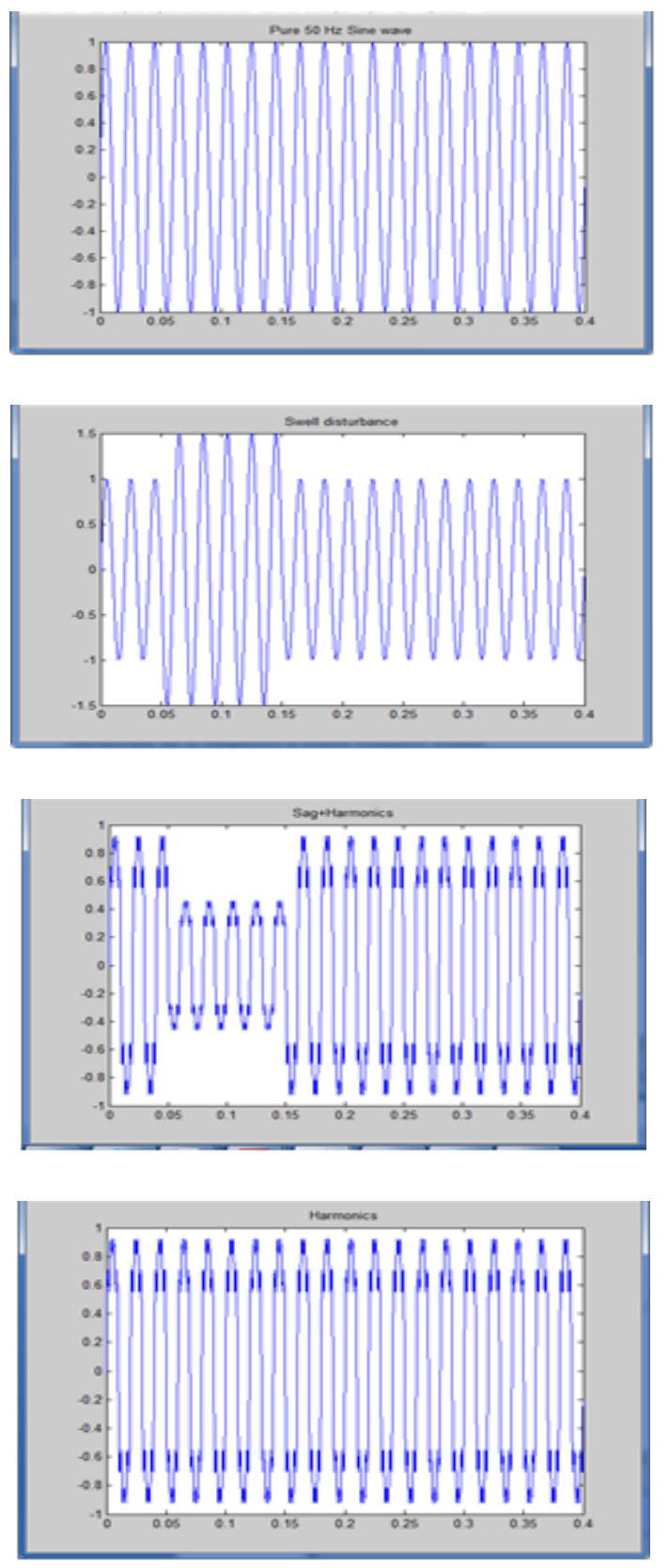

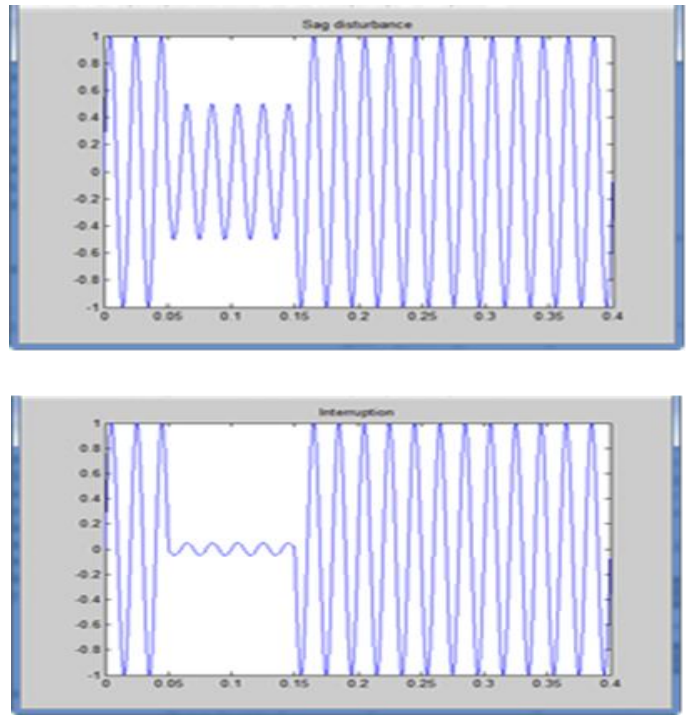

Fig .2. Different Types of PQ Disturbances

Multiresolution analysis leads to a hierarchical and fast schema and this can be implemented by a set of successive filter banks until the fourth level of decomposition and then reconstruction of the signal as we have done here in this algorithm. One approximation level which contains the fundamental frequency component and 4 detail levels that is the high frequency component which shows the abrupt change it the discontinuities to show the start and the end point of the disturbance as shown in fig. 3 for a sample of disturbance "voltage sag disturbance".

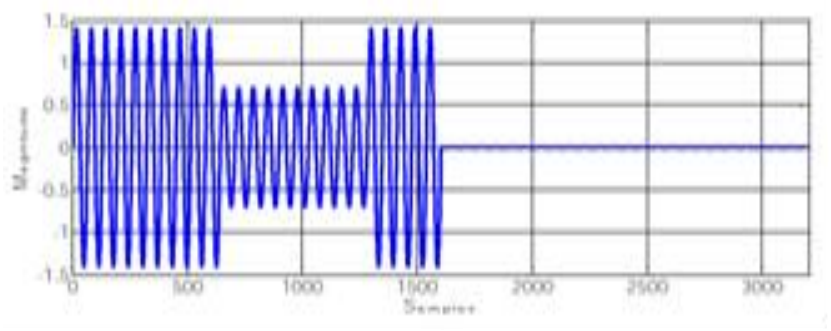

Decomposed voltage sag level 1 using WT

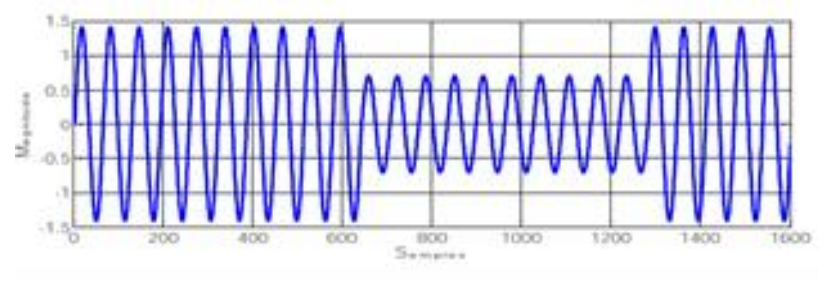

Approximate Signal level 1

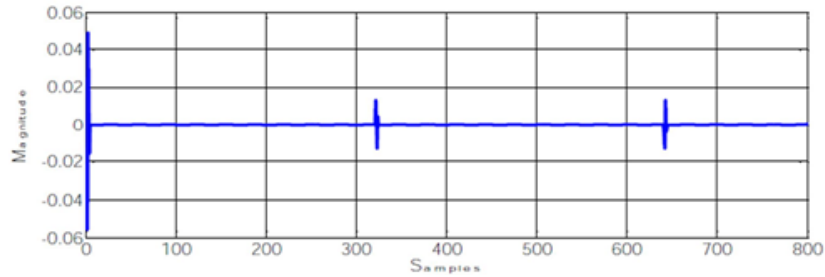

Detail Signal level 1

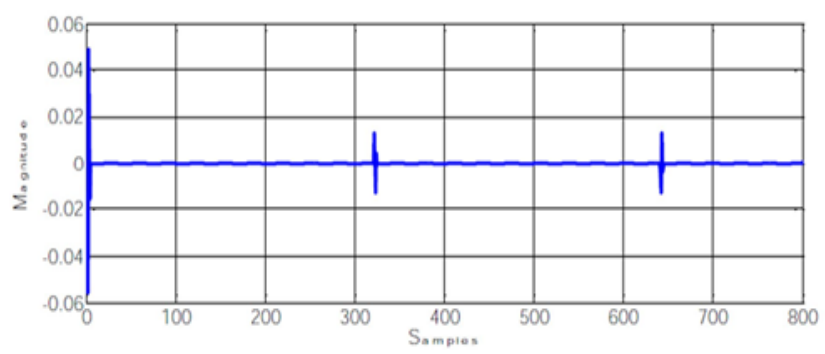

Detail Signal level 2

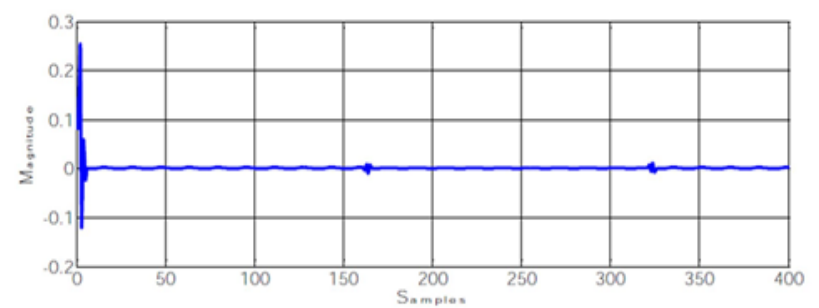

Detail signal level 3

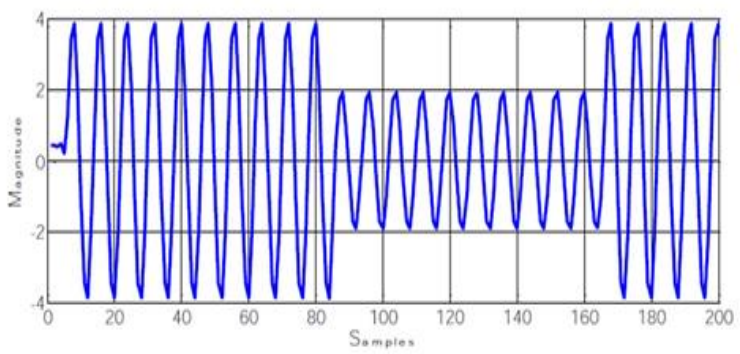

Approximate signal level 4

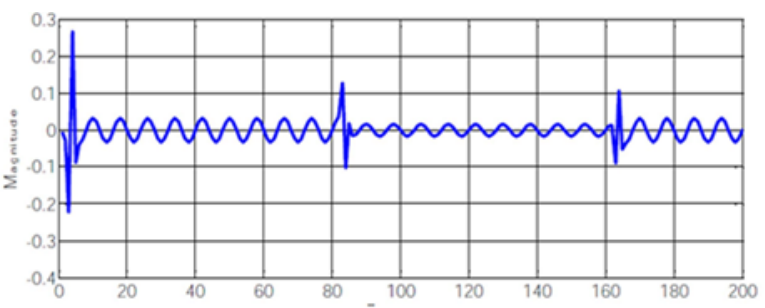

Detail signal level 4

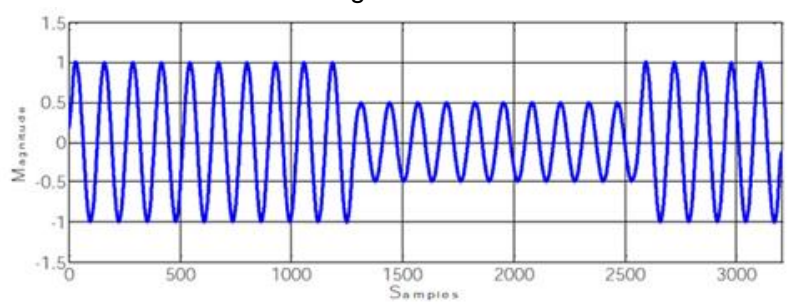

Reconstructed approximate signal 


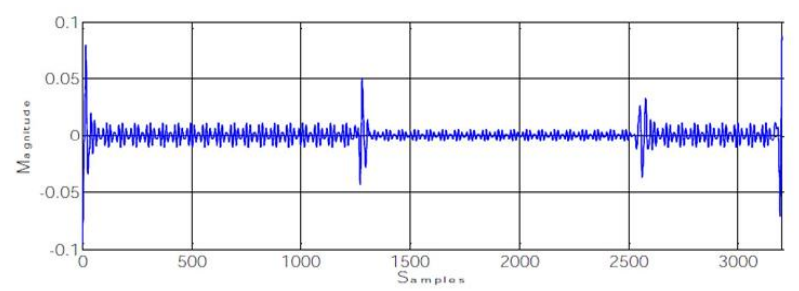

Reconstructed detail signal

Fig. 3. Feature Detection of a disturbance using the wavelet transform for the voltage sag

The norm-entropy based feature extraction based on the measure of the irregularities of states such as the uncertainty or the irregularity of the signal.

The norm entropy values for detail and approximation coefficients in each decomposition

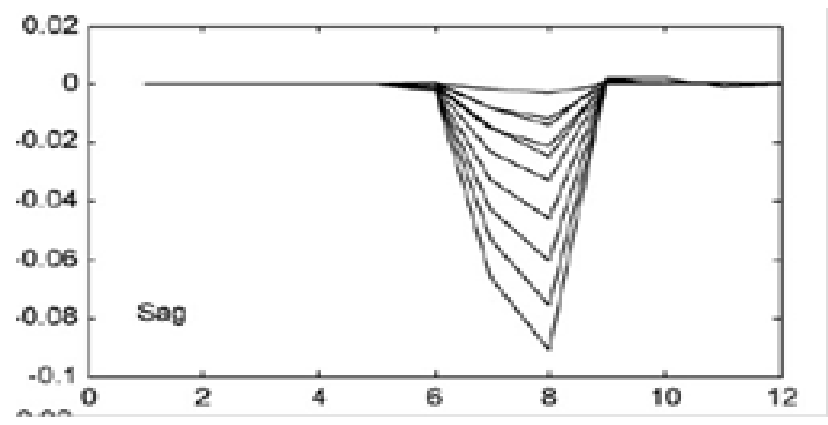

level from wavelet transform for different disturbances calculated as follows:

$$
\begin{gathered}
W_{d}=\left.\frac{1}{N_{j}} \sum_{n}\left|d_{j}[n] \|^{P} \quad W_{a j}=\frac{1}{N_{j}} \sum_{n}\right| a_{j}[n]\right|^{P} \\
(\mathrm{j}=1,2, \ldots, \mathrm{j})
\end{gathered}
$$

The wavelet Entropy Wsignal is a positive real number, $\mathrm{j}$ is the number of resolution levels. And the pure signal is used as a reference to distinguish disturbances from each other so we subtract the wavelet entropy of the pure sine of the wavelet entropy of the disturbance and as declared in fig.

Fig .4. The norm Entropy for some PQ disturbances

$$
\Delta \mathrm{W}=\mathrm{W}_{\text {disturbed }} \text { signal }-\mathrm{W}_{\text {pure signal }}
$$

DWT based decomposition is applied in for different PQ disturbances such as Sag, Swell, Interruption, Sag with harmonics and Swell with harmonics are generated using MATLAB and then decomposed using decomposition algorithm of WT and type of disturbance is detected.

\section{B. Classification using the fuzzy clustering technique}

The fuzzy clustering technique is based on two main blocks the first one is the wavelet feature extraction which work by extracting the different feature through the wavelet transform as declared in the previous section then the second block which the fuzzy inference system that maximize the accuracy of the detection and classifying the $P Q$ disturbances into clusters as the decision boundaries of disturbances may overlap we need to detect and classify the events overlap by the setting the fuzzy rules. Identifying the features of the membership function that used to identify the disturbances using the least number of features achieving the effective classification of $P Q$ events.

The rule of the artificial intelligence technique (Fuzzy Logic) is a measure of the degree of similarity of $P Q$ disturbances and the total distorted signal energy of the signal is compared to the corresponding pure signal. As mentioned above the Fuzzy logic block consists of the fuzzy inputs which are the feature extracted with the wavelet transform which are the norm entropy and the power factor and the Peak hour table and forming the fuzzy rules as follows :-

Considering the range for Power factor is from 0 to 1 and the values are high, medium and low, Rush hour

values are Peak as $\mathrm{P}$ or moderate $\mathrm{M}$ or low $\mathrm{L}$.

Rule 1:- if norm entropy is medium and Power factor is high and Rush hour is $L$ Then the $P Q$ disturbance is Normal pure signal 
Rule 2:- if norm entropy is low and Power factor is high and Rush hour is $L$ Then the $P Q$ disturbance is sag.

Rule 3:- if norm entropy is high and Power factor is high and Rush hour is $\mathrm{H}$ Then the $P Q$ disturbance is swell.

Rule 4:- if norm entropy is medium and Power factor is medium and Rush hour is $H$ Then the $P Q$ disturbance is harmonic.

Rule 5:- if norm entropy is low and Power factor is medium and Rush hour is $L$ Then the $P Q$ disturbance is sag + harmonics.

Rule 6:- if norm entropy is high and Power factor is medium and Rush hour is $\mathrm{H}$ Then the $\mathrm{PQ}$ disturbance is swell + harmonics.

Rule 7:- if norm entropy is low and Power factor is low and Rush hour is $H$ Then the $P Q$ disturbance is Interrupt.

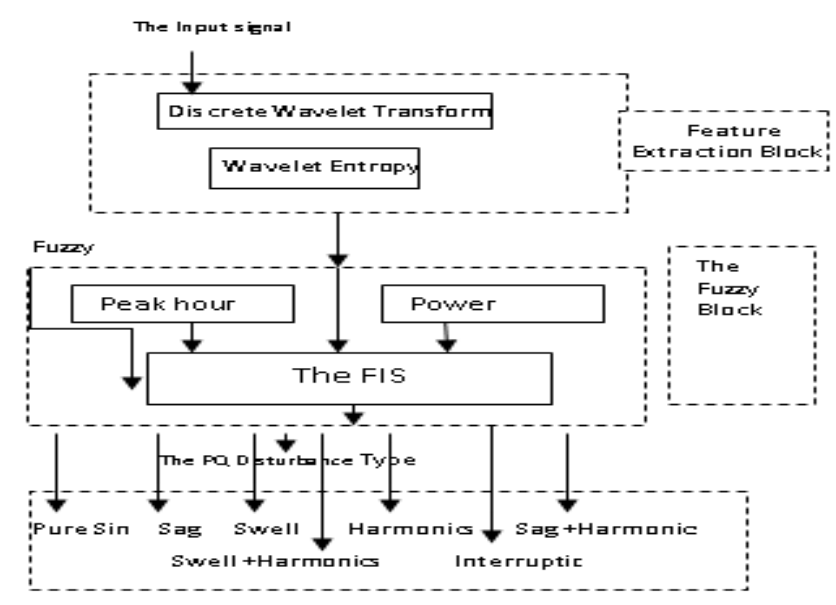

Fig. 5. The structure of $P Q$ Disturbance Classification based on the WT and the fuzzy Logic

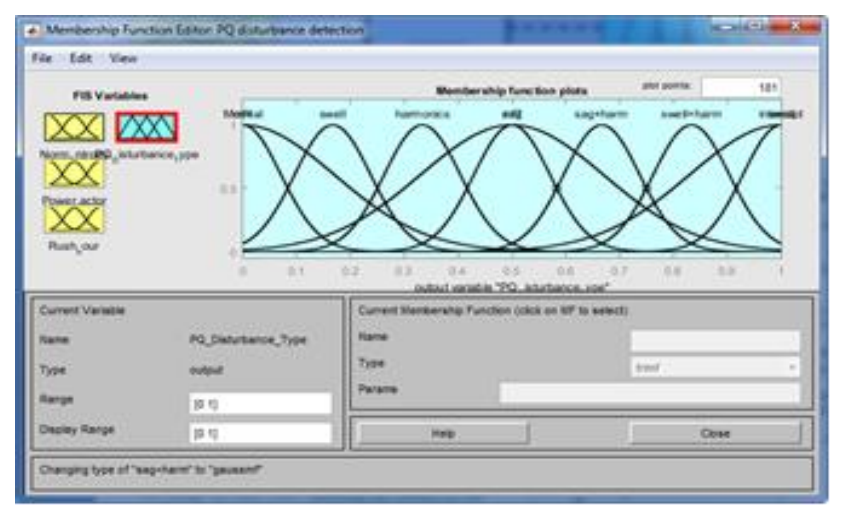

Fig .6. The FIS For the membership functions of the $P Q$ disturbance detection
In the FL algorithm we used 100 signal for detection and classification and the classification result is shown in table 1 using the parameters of the power factor and the peak hour table so the fuzzy logic is able to detect and classify the power quality disturbance accurately.

\section{Classification through Multilayer Feed forward Neural Network (MFNN)}

Neural networks are simplified model of the biological nervous system as it's a highly interconnected network of a large number of processing elements (PEs) called neurons that composes the topology of the Artificial Neural Network (ANN) and the learning process is done by being trained on some examples by giving data to acquire knowledge about the problem, the neural networks adopt various learning mechanisms of supervised and unsupervised learning [8].

Artificial Neural Network are characterized by the number of

interconnections and the node characteristics that are classified by the kind of learning rules has been employed and the type of non-linear elements has been used.

Artificial Neural Networks (ANN) approaches have been developed to solve problems in power plants and power systems using the process identification, sensor validation, monitoring and fault diagnosis, in power plants, and security assessment, load identification, load modeling, forecasting and fault diagnosis, in power systems.

As in our algorithm using the wavelet and neural network combine the aspects of the wavelet transformation which works on the frequency domain for the purpose of feature extraction and selection and the characteristic decision capabilities of neural network approaches.

Probabilistic Neural Network for classification of $P Q$ events based on the features extracted from the detail coefficients through decompositions.

We used an artificial neural network with 3 layers as the inputs are the feature extracted using the wavelet transform (feature extraction block) and the output specifies the class of disturbance using multiple instances of the original waveform signal as a test 
pattern and multiple instances of the various types of disturbances as a training set for the neural networks.

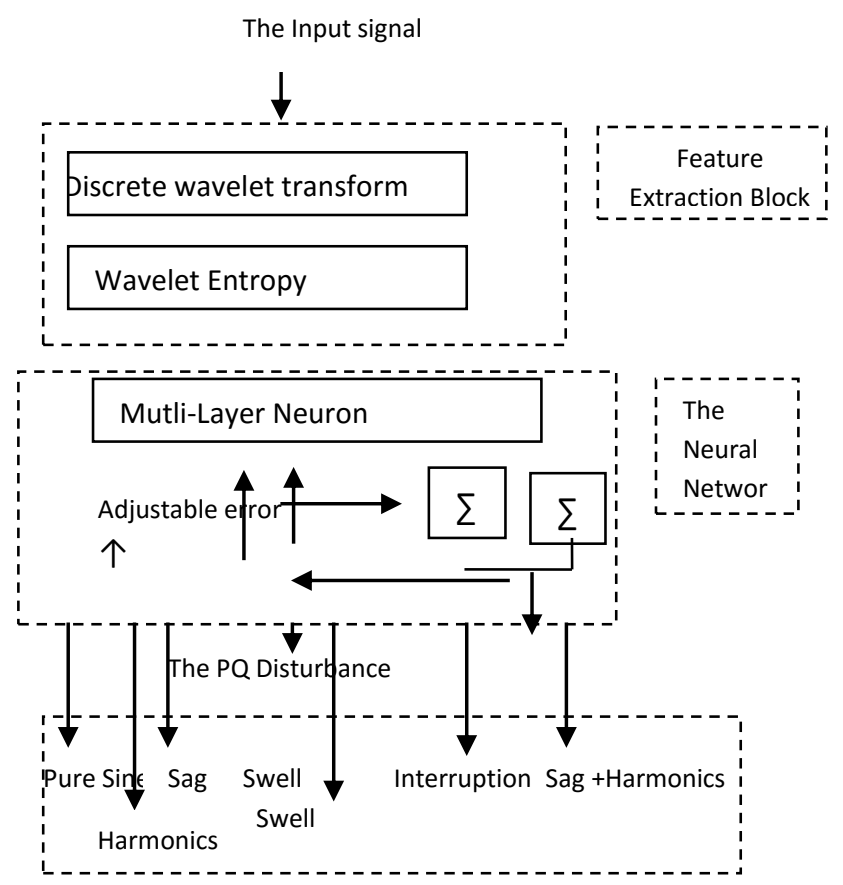

Fig. 7.Architecture of the PQ Disturbance Classification Using WT and ANN

The structure of the network is a 3- layered neural ne network architecture with sigmoid activation function and error back propagation for supervised learning as the inputs are the features extracted from wavelet transform and other factors feed like the density of the feeding nodes and its distribution also the power factor and the peak hour table and the 7 classes of power quality disturbances as the output of the NN and 100 signal is used for training the network to be adjusted according to its error and another 100 for testing the algorithm to measure the network performance after training.

\section{SIMULATION RESULTS AND EVALUATION}

The classification was done with power quality disturbance signals simulated by MATLAB version R2015a. The classes include the voltage sag, voltage swell, harmonics, sag with harmonics, swell with harmonics and the interrupt.
Table 1. CLASSIFICATION RESULT

\begin{tabular}{|l|c|c|}
\hline \multirow{2}{*}{ CLASS } & \multicolumn{2}{|c|}{ CLASSIFICATION \% } \\
\cline { 2 - 3 } & FUZZY LOGIC & $\begin{array}{l}\text { ARTIFICIAL NEURAL } \\
\text { NETWORK } \\
\text { SUBHEAD }\end{array}$ \\
\hline $\begin{array}{l}\text { SAG } \\
\text { DISTURBANCE }\end{array}$ & 98 & 97 \\
\hline $\begin{array}{l}\text { SWELL } \\
\text { DISTURBANCE }\end{array}$ & 97 & 96 \\
\hline $\begin{array}{l}\text { HARMONIC } \\
\text { DISTURBANCE }\end{array}$ & 94 & 95 \\
\hline $\begin{array}{l}\text { HARMONIC+ } \\
\text { SAG }\end{array}$ & 95 & 96 \\
\hline $\begin{array}{l}\text { HARMONIC+ } \\
\text { SWELL }\end{array}$ & 94 & 95 \\
\hline INTERRUPT & 98 & 97 \\
\hline
\end{tabular}

The results proved that the two systems are effective in detecting the $P Q$ disturbances but in the neural network algorithm there is no rule to determine the data required for the training of each disturbance types and if more data available for training it will give better accuracy but will increase time to train the network And the rules of the fuzzy technique not adaptable according to the variation in data.

\section{CONCLUSION}

In this paper we proposed two algorithms for the fast and accurate detection of the power quality disturbance detection based on the intelligent computing techniques and the Multi-resolution analysis. The algorithms are the fuzzy logic and the neural network and both of them depends mainly on detecting the features using the wavelet transform.

Fuzzy logic system has strong inference capabilities of expert system as well as power of natural knowledge representation. But the rules are based on the human experience and expertise. A membership function provides a measure of the degree of similarity of an element in the fuzzy subset. And we can't modify the membership function. So The rules of the fuzzy algorithm should be updated and weighting factors in the fuzzy sets should be refined by using the Neural networks.

In the artificial neural network the classification accuracy increases by increasing the number of inputs applied to it but as the architecture of the neural network is getting more complex the computational complexity increases too. 
We might think of using a hybrid system incorporating the learning capabilities of ANN and excellent knowledge representation and inference capabilities of fuzzy logic that have the ability to self modify their membership function to achieve the desired performance.

\section{REFERENCES}

[1] Rafael E. Bourguet and Panos J. Antsaklis,"Artificial Neural Networks In Electric Power Industry", Technical Report of the ISIS Group at the University of Notre Dame, ISIS-94007 April, 1994

[2] Min Wang, Piotr ochenkowski, Alexander Mamishev, "Classification of power quality disturbances using time-frequency ambiguity plane and neural networks", 2001 IEEE .

[3] Saxena, K.S. Verma, and S.N. Singh, "Power quality event classification: an overview and key issues",2010 .

[4] Mahmoud El-Gammal, Amr Abou-Ghazala and Tarek El-Shennawy," Detection, localization, and classification of power quality disturbances using discrete wavelet transform technique",Alexandria Engineering Journal 2003.
[5] Mohamed E.Salem Abozaed,"Detection and classification of power quality disturbances using S-Transform and wavelet algorithm", International Journa; of Electrical , Computer,Energetic, Electronic and communication Engineering Vol7, No:6, 2013.

[6] S.Suja,Jovitha Jerome, "Power signal disturbance clssification using wavelet based neural network",Serbian journal of electric engineering, Vol. 4, No. 1,June 2007,71-83.

[7] Pearson,"Fuzzy Logic Fundamentals”, Chapter3 , 26 March 2001.

[8] S.Rajasekaran, G.A.vijayalakshami, "Neural networks, fuzzy logic and genetic algorithm: synthesis and applications, july 2011.

[9] Zahir Javed paracha ,Design and development of intelligent computational technique for power quality data monitoring and management", Victoria university Australia, 2011.

[10] Janusz Mindykowski, "Fundamentals of electric power assessment", Precedings ,XVII IMEKO world congress, Uune 22-27 2003, Dubrovnik, Coroatia.

[11] Zadeh, L.A. (1965): Fuzzy sets, Information and Control 8(3):338-353. 